\title{
BOUNDARY VALUE PROBLEMS OF THE CALCULUS OF VARIATIONS $\dagger$
}

\author{
BY W. T. REID
}

1. Introduction. The term boundary value problem is applied to the question of determining whether a given system of differential equations (in general, a given system of functional equations) has one or more solutions satisfying certain prescribed end or boundary conditions; and, if so, the determination of the character of these solutions and how their character changes when the differential equations or boundary conditions change. This address is restricted to the discussion of a class of linear boundary problems which are intimately associated with the calculus of variations. These boundary value problems have been used in establishing sufficient conditions, especially for the more complicated problems of the calculus of variations. On the other hand, the principles and theorems of the calculus of variations have been of extreme significance in the advancement of the theory of such boundary value problems. In fact, the calculus of variations has served to unify a certain class of boundary problems much larger than that seemingly represented by the problem that we shall first formulate. In view of the rather extensive interest and study of these problems within recent years, it seems proper at this time to discuss the present status of such boundary problems, to compare the various methods that have been used in their treatment, and to indicate various questions concerning them that are as yet unsolved.

Historically, the study of boundary problems associated with a second order linear differential equation dates from the time of Euler and D'Alembert. The first somewhat general theory of such problems, however, is that given by Sturm [1] $\ddagger$ in his fundamental memoir of 1836. One of the most important questions for the more complicated boundary problems that we shall here consider is that of generalizing the Sturm oscillation and

$\dagger$ An address delivered by invitation of the program committee at the Chicago meeting of this Society, April 9, 1937.

$\ddagger$ Numerals in square brackets refer to the bibliography at the end of the paper. 
comparison theorems for a single second order equation. Consequently, I shall describe them briefly. Perhaps the most fundamental result of Sturm's paper is the following comparison theorem:

Suppose $u_{1}$ and $u_{2}$ are solutions of the equations

$$
\begin{aligned}
& \left(K_{1} u_{1}^{\prime}\right)^{\prime}-G_{1} u_{1}=0, \\
& \left(K_{2} u_{2}^{\prime}\right)^{\prime}-G_{2} u_{2}=0,
\end{aligned}
$$

where $K_{1} \geqq K_{2}>0, G_{1} \geqq G_{2}$ and both equality signs do not hold throughout $x_{1} x_{2}$, while $u_{1}\left(x_{1}\right)=0=u_{1}\left(x_{2}\right)$. Then $u_{2}$ vanishes at least once on $x_{1}<x<x_{2}$.

The general oscillation theorem of Sturm's paper is concerned with a differential equation

$$
\left(K(x, \lambda) u^{\prime}\right)^{\prime}-G(x, \lambda) u=0
$$

with two-point boundary conditions of the form

$$
\begin{aligned}
& \alpha_{1}(\lambda) u\left(x_{1}\right)-\beta_{1}(\lambda) K\left(x_{1}, \lambda\right) u^{\prime}\left(x_{1}\right)=0, \\
& \alpha_{2}(\lambda) u\left(x_{2}\right)+\beta_{2}(\lambda) K\left(x_{2}, \lambda\right) u^{\prime}\left(x_{2}\right)=0 .
\end{aligned}
$$

Under certain general conditions, which include in particular the assumption that $K(x, \lambda), G(x, \lambda), \alpha_{1}(\lambda) / \beta_{1}(\lambda)$, and $\alpha_{2}(\lambda) / \beta_{2}(\lambda)$ are monotone decreasing functions of $\lambda$, it is established that there exists an infinity of characteristic values $\lambda_{1}<\lambda_{2}<\cdots<\lambda_{n}<\cdots$, and that the characteristic solution $u_{n}$ corresponding to $\lambda_{n}$ has exactly $n-1$ zeros on $x_{1}<x<x_{2}$. It is to be noted that (2) is the Euler equation and (3) the transversality conditions associated with the functional

$$
\begin{aligned}
{\left[\alpha_{1}(\lambda) / \beta_{1}(\lambda)\right] u^{2}\left(x_{1}\right) } & +\left[\alpha_{2}(\lambda) / \beta_{2}(\lambda)\right] u^{2}\left(x_{2}\right) \\
& +\int_{x_{1}}^{x_{2}}\left[K(x, \lambda) u^{\prime 2}+G(x, \lambda) u^{2}\right] d x .
\end{aligned}
$$

By way of introduction, consider the problem of minimizing the integral

$$
J=\int_{x_{1}}^{x_{2}} f\left(x, y, y^{\prime}\right) d x
$$

in the class of arcs $y$ of the form $y=y(x)$ on $x_{1} \leqq x \leqq x_{2}$ and joining the two points $P_{1}:\left(x_{1}, y_{1}\right)$ and $P_{2}:\left(x_{2}, y_{2}\right)$. Along a minimizing arc $E$ the second variation 


$$
J_{2}=\int_{x_{1}}^{x_{2}}\left(f_{r r} \eta^{\prime 2}+2 f_{r y} \eta^{\prime} \eta+f_{y y} \eta^{2}\right) d x
$$

must be non-negative in the class of arcs $\eta=\eta(x), x_{1} \leqq x \leqq x_{2}$, such that $\eta\left(x_{1}\right)=0=\eta\left(x_{2}\right)$. Moreover, for the given arc $E$ to be a minimizing arc it must be true that $f_{r r} \geqq 0$ along $E$. If we suppose that $E$ is an extremal which is non-singular in the sense that along it we have $f_{r r} \neq 0$, then the usual Jacobi necessary condition states that there exists no non-identically vanishing solution $\eta(x)$ of the Jacobi equation

$$
J(\eta) \equiv\left(f_{r r} \eta^{\prime}+f_{r y} \eta\right)^{\prime}-\left(f_{r y} \eta^{\prime}+f_{y y} \eta\right)=0
$$

such that $\eta\left(x_{1}\right)=0=\eta\left(x_{3}\right)$, and $x_{1}<x_{3}<x_{2}$. A point $x_{3}$ for which such a solution of (7) exists is said to determine a point $P_{3}:\left[x_{3}, y\left(x_{3}\right)\right]$ on $E$ conjugate to $P_{1}$. If the strengthened Jacobi condition is satisfied, that is, if there is no value $x_{3}$ on $x_{1}<x_{3} \leqq x_{2}$ determining a point conjugate to $P_{1}$, the second variation $J_{2}[\eta]$ may be shown to be positive for all non-identically vanishing arcs.

Now consider the boundary problem

$$
J(\eta)+\lambda \eta=0, \quad \eta\left(x_{1}\right)=0=\eta\left(x_{2}\right)
$$

involving the characteristic parameter $\lambda$. A value $\lambda$ is called a characteristic value of (8) if there exists a corresponding nonidentically vanishing solution $\eta$ of (8). One may show in a simple manner that all the characteristic values of (8) are real. If $\lambda$ is a characteristic value of (8) and $\eta$ a corresponding solution, it follows by integration by parts that

$$
J_{2}[\eta]=\lambda \int_{x_{1}}^{x_{2}} \eta^{2} d x .
$$

Hence in order that $J_{2} \geqq 0$ it is necessary that there exist no negative characteristic values of this system. Clearly $x_{2}$ determines $P_{2}$ conjugate to $P_{1}$ on $E$ if and only if $\lambda=0$ is a characteristic value of (8).

It is a consequence of Sturm's oscillation and comparison theorems that the Jacobi condition is equivalent to the condition that there exist no negative characteristic values of (8). Furthermore, if one utilizes expansion theorems for arbitrary functions $\eta(x)$ satisfying the end-conditions in terms of the char- 
acteristic solutions of (8), it may be shown that the smallest characteristic value of this system is actually the minimum of $J_{2}[\eta]$ in the class of $\eta$ 's satisfying the end conditions and normed so that $\int_{x_{1}}^{x_{2}} \eta^{2} d x=1$. Now by the introduction of the Green's function the system (8) is seen to be equivalent to an integral equation with real symmetric kernel, and such an expansion theorem follows from the Hilbert-Schmidt theory of integral equations. Indeed, it is a ready consequence of the expansion theorem that each characteristic value of $(8)$ is the minimum value of $J_{2}[\eta]$ in a suitable class of $\operatorname{arcs} \eta$.

It is to be emphasized that the choice of the associated boundary value problem is somewhat arbitrary. In the above case one might equally well have considered the problem

$$
J(\eta)+\lambda k(x) \eta=0, \quad \eta\left(x_{1}\right)=0=\eta\left(x_{2}\right)
$$

where $k(x)$ is a positive function on $x_{1} x_{2}$. It is also to be noted that for an admissible arc $\eta$ the second variation reduces on integration by parts to

$$
\mathfrak{J}_{2}[\eta]=\int_{x_{1}}^{x_{2}}\left(f_{r r}(x) \eta^{\prime 2}+g \eta^{2}\right) d x, \quad\left(g=f_{y y}-d f_{y r} / d x\right) .
$$

If one considers the boundary problem

$$
\left(f_{r r} \eta^{\prime}\right)^{\prime}-\lambda g \eta=0, \quad \eta\left(x_{1}\right)=0=\eta\left(x_{2}\right),
$$

it may be shown that $J_{2} \geqq 0(>0)$ if and only if the smallest positive characteristic value is $\geqq 1(>1)$. This form of the associated boundary value problem has been used by Lichtenstein for both simple and double integral problems of the calculus of variations.

For the more complicated problems of the calculus of variations one may associate with the second variation a linear boundary problem in a manner analogous to that indicated above for the plane problem. Such a formulation for the general problem of Mayer was first explicitly given by Cope [1]. Immediately there arises the question of the existence of characteristic values for this problem, and the relations between such a boundary problem and the positiveness of the second variation. Can one generalize the results of the Sturmian theory to this more general boundary problem and thus prove the existence of characteristic values and properties of correspond- 
ing solutions? Actually, this order of proof has been carried through only recently. For boundary value problems associated with calculus of variations problems of varying complexity the existence of characteristic values has been established by various methods. Among the methods which have been used are the following, and various combinations of them: (1) the use of comparison and approximation theorems, (2) asymptotic expressions, (3) integral equations, (4) approximating algebraic difference equations, (5) the theory of linear algebraic equations in an infinite number of variables, (6) general theorems on differential equations, (7) the minimum principle of the calculus of variations.

From the standpoint of comparison and oscillation theorems for the more general boundary problems, it is to be remarked that either these theorems may first be proved and the existence of characteristic values deduced from them, or the existence of characteristic values may be first proved and these comparison and oscillation theorems then proved as a consequence of the extremizing properties of the characteristic values and solutions.

2. Formulation of a General Boundary Problem. Bliss [6] has emphasized the significant position of the so-called problem of Bolza with respect to the other problems of the calculus of variations. For brevity, the general problem of Bolza will not be formulated here. We shall, however, give the form of the second variation of such a problem, and discuss associated boundary value problems. The symbols $\eta \equiv\left[\eta_{i}\right], \eta^{\prime} \equiv\left[\eta_{i}^{\prime}\right]$ will denote the functions $\left[\eta_{1}(x), \cdots, \eta_{n}(x)\right]$ and the set of their derivatives, respectively. The second variation for a problem of Bolza may then be written

$$
J_{2}[\eta] \equiv 2 Q\left[\eta\left(x_{1}\right), \eta\left(x_{2}\right)\right]+\int_{x_{1}}^{x_{2}} 2 \omega\left(x, \eta, \eta^{\prime}\right) d x,
$$

where $\omega$ and $Q$ are quadratic forms in the $2 n$ variables $\eta_{i}, \eta_{i}^{\prime}$ and $\eta_{i}\left(x_{1}\right), \eta_{i}\left(x_{2}\right)$, respectively. The associated equations of variation of the auxiliary differential and end-conditions of the problem of Bolza are of the form

$$
\begin{array}{r}
\Phi_{\alpha}\left(x, \eta, \eta^{\prime}\right) \equiv \Phi_{\alpha \pi_{i}}(x) \eta_{i}^{\prime}+\Phi_{\alpha \eta_{i}}(x) \eta_{i}=0, \\
(\alpha=1, \cdots, m<n),
\end{array}
$$




$$
\begin{aligned}
\Psi_{\gamma}\left[\eta\left(x_{1}\right), \eta\left(x_{2}\right)\right] \equiv \Psi_{\gamma ; i 1} \eta_{i}\left(x_{1}\right)+\Psi_{\gamma ; i 2} \eta_{i}\left(x_{2}\right) & =0, \\
& (\gamma=1, \cdots, p \leqq 2 n) .
\end{aligned}
$$

It is to be remarked that in order to write the second variation of a problem of Bolza in the form (10) it is in general necessary to include in the set $\eta$ not only the variations of the dependent functions in the original problem of Bolza, but also two new functions representing the variations of the end-values; these latter two functions are further restricted by including in the set (11) two additional differential equations which require them to be constant on $x_{1} x_{2}$ (see, for example, Reid [2]).

Partial derivatives of $\omega(x, \eta, \pi), \Phi_{\alpha}(x, \eta, \pi)$ with respect to the variables, $\eta_{i}, \pi_{i}$ will be denoted by writing these variables as subscripts; derivatives of $Q$ and $\Psi_{\gamma}$ with respect to the arguments $\eta_{i}\left(x_{1}\right), \eta_{i}\left(x_{2}\right)$ will be indicated by $Q_{i 1}, \Psi_{\gamma ; i 1}, Q_{i 2}, \Psi_{\gamma ; i 2}$, respectively. The summation convention of tensor analysis is used throughout.

The conditions usually required of an arc for the problem of Bolza in order to obtain sufficiency theorems insure for the second variation the following properties.

$\left(\mathrm{H}_{1}\right)$ The coefficients of the quadratic form $\omega(x, \eta, \pi)$ and the linear expressions $\Phi_{\alpha}(x, \eta, \pi)$ are real single-valued functions of class $C^{1}$, and the matrix $\left\|\Phi_{\alpha \pi_{j}}(x)\right\|$ has rank $m$ on $x_{1} x_{2}$. The coefficients of $Q$ and $\Psi_{\gamma}$ are real constants, and the matrix $\left\|\Psi_{\gamma ; i 1} \Psi_{\gamma ; i 2}\right\|$ is of rank $p$.

$\left(\mathrm{H}_{2}\right)$ The quadratic form $\omega_{\pi_{i} \pi_{j}}(x) u_{i} u_{j}$ is positive for all values $\left(u_{i}\right) \neq\left(0_{i}\right)$ satisfying $\Phi_{\alpha \pi_{j}}(x) u_{j}=0,(\alpha=1, \cdots, m)$.

Condition $\left(\mathrm{H}_{2}\right)$ implies, in particular, that the matrix

$$
\left|\begin{array}{cc}
\omega_{\pi_{i} \pi_{j}} & \Phi_{\alpha \pi_{i}} \\
\Phi_{\beta \pi_{j}} & 0_{\beta \alpha}
\end{array}\right|, \quad(i, j=1, \cdots, n ; \beta, \alpha=1, \cdots, m)
$$

is non-singular on $x_{1} x_{2}$.

An arc $\eta \equiv\left[\eta_{i}(x)\right]$ will be called differentially admissible if the functions $\eta_{i}(x)$ are of class $D^{1}$ and satisfy the equations (11) on $x_{1} x_{2}$. An arc whose end values satisfy equations (12) will be said to be terminally admissible. Finally, an arc which is both differentially and terminally admissible will, for brevity, be termed admissible.

$\left(\mathrm{H}_{3}\right)$ There exist $p$ differentially admissible arcs $\eta_{i}=\eta_{i v}(x)$, 
$(\nu=1, \cdots, p)$, such that the determinant $\left|\Psi_{\gamma}\left[\eta_{\nu}\left(x_{1}\right), \eta_{\nu}\left(x_{2}\right)\right]\right|$ is different from zero.

Hypothesis $\left(\mathrm{H}_{3}\right)$ is a condition of normality with respect to the differential equations (11) and end conditions (12).

The accessory (Jacobi) system along a given non-singular extremal for the original problem of Bolza is defined as the EulerLagrange equations and transversality conditions for the corresponding second variation (10) and the auxiliary differential equations (11). If we set $\Omega(x, \eta, \pi, \mu)=\omega(x, \eta, \pi)+\mu_{\alpha} \Phi_{\alpha}(x, \eta, \pi)$, these differential equations are

$$
\begin{aligned}
J_{i}(\eta, \mu) \equiv d \Omega_{\pi_{i}} / d x-\Omega_{\eta_{i}}=0, \quad \Phi_{\alpha} & =0, \\
(i=1, \cdots, n ; \alpha & =1, \cdots, m) ;
\end{aligned}
$$

the corresponding transversality conditions involve constants $d_{\gamma}$ satisfying with the end values of $\eta_{i}, \mu_{\alpha}$ the relations

$$
\begin{aligned}
Q_{i s}[\eta]+d_{\gamma} \Psi_{\gamma_{i} i s}+(-1)^{s} \Omega_{\pi_{i}}\left(x_{s}\right) & =0, \quad \Psi_{\gamma}=0, \\
(s & =1,2 ; \gamma=1, \cdots, p) .
\end{aligned}
$$

The associated accessory boundary problem consists of the equations

$$
J_{i}(\eta, \mu)+\lambda \eta_{i}=0, \quad \Phi_{\alpha}=0
$$

involving the characteristic parameter $\lambda$, together with the end conditions (15). A value $\lambda$ will be said to be a characteristic value of this problem if there exist corresponding solutions $\eta_{i}, \mu_{\alpha}$ of (16) not all identically zero, and whose end values at $x_{1}$ and $x_{2}$ satisfy with suitable constants $d_{\gamma}$ the end-conditions (15). The above hypothesis $\left(\mathrm{H}_{3}\right)$ insures the condition $\eta_{i} \eta_{i} \neq \equiv$ on $x_{1} x_{2}$ for an arbitrary solution $\eta_{i}, \mu_{\alpha}$ of this system. By a method similar to that indicated in the introduction for the simple problem of the calculus of variations, one may show that in order for $J_{2}[\eta]$ to be non-negative in the class of admissible arcs $\eta$ it is necessary that there exist no negative characteristic value of (16), (15). In view of the minimizing properties of the characteristic values of this system to be discussed later, one may also state that the non-existence of negative characteristic values of the accessory problem is equivalent to the non-negativeness of $J_{2}[\eta]$ in the class of admissible arcs $\eta$.

Since the matrix (13) is assumed to be non-singular, canonical variables $\zeta_{i}$ may be introduced by means of the equations 


$$
\begin{array}{r}
\zeta_{i}=\Omega_{\pi_{i}}(x, \eta, \pi, \mu), \quad 0=\Phi_{\alpha}(x, \eta, \pi), \\
(i=1, \cdots, n ; \alpha=1, \cdots, m),
\end{array}
$$

and system (16) may be written as

$$
\eta_{i}^{\prime}=A_{i j}(x) \eta_{j}+B_{i j}(x) \zeta_{j}, \zeta_{i}^{\prime}=C_{i j}(x) \eta_{j}-A_{j i}(x) \zeta_{j}-\lambda \eta_{i} .
$$

The elements $A_{i j}, B_{i j}, C_{i j}$ in (17) are continuous, the matrices $\left\|B_{i j}\right\|,\left\|C_{i j}\right\|$ are symmetric, and $\left\|B_{i j}\right\|$ is of rank $n-m$ on $x_{1} x_{2}$. Correspondingly, the end conditions (15) reduce to

$$
\begin{aligned}
s_{\sigma}[\eta, \zeta] \equiv a_{\sigma i} \eta_{i}\left(x_{1}\right)-b_{\sigma i} \zeta_{i}\left(x_{1}\right)+c_{\sigma i} \eta_{i}\left(x_{2}\right) & +d_{\sigma i} \zeta_{i}\left(x_{2}\right), \\
& (\sigma=1, \cdots, 2 n),
\end{aligned}
$$

where the coefficients of $s_{\sigma}$ are such that the matrix $\left\|a_{\sigma i} b_{\tau i}+c_{\sigma i} d_{r i}\right\|$, $(\sigma, \tau=1, \cdots, 2 n)$, is symmetric. Moreover, for an arbitrary system of the form (17), (18) whose coefficients satisfy the above conditions there exist quadratic forms $Q\left[\eta\left(x_{1}\right), \eta\left(x_{2}\right)\right], \omega\left(x, \eta, \eta^{\prime}\right)$ with associated linear equations $\Phi_{\alpha}\left(x, \eta, \eta^{\prime}\right)=0,(\alpha=1, \cdots, m)$, and end conditions $\Psi_{\gamma}=0$ such that the given system is derived from this associated system (10), (11), (12) in the manner described above (see $\mathrm{Hu}[1]$ ).

It is to be noted that the equations (16), (15) are the Euler. Lagrange equations and transversality conditions for the functional

$$
J_{2}[\eta]-\lambda \int_{x_{1}}^{x_{2}} \eta_{i} \eta_{i} d x
$$

subject to the differential equations (11) and end-conditions (12). The more general boundary problem to be considered consists of these equations for a functional

$$
\Im_{2}[\eta: \lambda]=2 Q\left[\eta\left(x_{1}\right), \eta\left(x_{2}\right): \lambda\right]+\int_{x}^{x_{2}} 2 \omega\left(x, \eta, \eta^{\prime}: \lambda\right) d x
$$

subject to (11) and (12). The canonical form of this problem is then

$$
\begin{gathered}
\text { (21) } \eta_{i}^{\prime}=A_{i j}(x: \lambda) \eta_{j}+B_{i j}(x: \lambda) \zeta_{j}, ! \zeta_{i}^{\prime}=C_{i j}(x: \lambda) \eta_{j}-A_{j i}(x: \lambda) \zeta_{j}, \\
s_{\sigma}[\eta, \zeta: \lambda]=0 .
\end{gathered}
$$

For the still more general problem in which the coefficients of (11) and (12) also depend on $\lambda$, at present no important re- 
sults concerning the existence of characteristic values and associated comparison and oscillation theorems have been obtained. In fact, one may show by simple examples that these conditions as usually phrased are in general not satisfied by such problems.

Of particular significance is the special problem of the form (21) in which this system reduces to

(22) $\eta_{i}^{\prime}=A_{i j}(x) \eta_{j}+B_{i j}(x) \zeta_{j}, \zeta_{i}^{\prime}=C_{i j}(x) \eta_{j}-A_{j i}(x) \zeta_{j}-\lambda K_{i j} \eta_{j}$

$$
s_{\sigma}[\eta, \zeta]=0 \text {, }
$$

that is, the case in which $Q$ is independent of $\lambda$ and $2 \omega\left(x, \eta, \eta^{\prime}: \lambda\right)$ $\equiv 2 \omega\left(x, \eta, \eta^{\prime}\right)-\lambda \eta_{i} K_{i j}(x) n_{i}$.

The above assumption $\left(\mathrm{H}_{3}\right)$ is equivalent to the condition that there is no characteristic solution $\eta_{i}, \zeta_{i}$ for which $\eta_{i} \equiv 0$ on $x_{1} x_{2}$ (see, for example, Bliss [4], p. 48). As is customary (Reid [4], p. 575), we shall say that the order of anormality on $x_{1} x_{2}$ of the differential equations of the above boundary problem is equal to $r$ if on this interval they possess exactly $r$ linearly independent solutions of the form $\eta_{i} \equiv 0, \zeta_{i}=v_{i h}(x),(h=1, \cdots, r)$. Since the expressions $\Phi_{\alpha}$ do not depend on $\lambda$, the value of $r$ is independent of $\lambda$. It follows readily that $r \leqq m$; moreover, if $\eta$ is an arbitrary differentially admissible arc and $x^{\prime} x^{\prime \prime}$ are any two points of $x_{1} x_{2}$, then

$$
\left.v_{i h}(x) \eta_{i}(x)\right|_{x^{\prime}} ^{x^{\prime \prime}}=0, \quad(h=1, \cdots, r) .
$$

As a consequence of these relations, hypothesis $\left(\mathrm{H}_{3}\right)$ is equivalent to the assumption that the matrix

$$
\begin{gathered}
\left\|\begin{array}{cc}
\Phi_{\gamma ; j 1} & \Psi_{\gamma ; j 2} \\
-v_{j h}\left(x_{1}\right) & v_{j h}\left(x_{2}\right)
\end{array}\right\|, \\
(j=1, \cdots, n ; h=1, \cdots, r ; \gamma=1, \cdots, p),
\end{gathered}
$$

has rank $p+r$. This implies, in particular, that $p \leqq 2 n-r$.

Now the class of admissible arcs for a problem is unchanged when the conditions $\Psi_{\gamma}=0$ are replaced by conditions of the form

$\Psi_{\gamma}+e_{\gamma h}\left[-v_{j h}\left(x_{1}\right) \eta_{j}\left(x_{1}\right)+v_{j h}\left(x_{2}\right) \eta_{j}\left(x_{2}\right)\right]=0, \quad(\gamma=1, \cdots, p)$, where $e_{\gamma h},(\gamma=1, \cdots, p ; h=1, \cdots, r)$, are arbitrary constants. If $\eta_{i}, \zeta_{i}$ is a characteristic solution of the original problem, there 
are constants $e_{h}$ such that $\eta_{i}, \zeta_{i}+v_{i h} e_{h}$ is a characteristic solution of the modified problem. Consequently, we do not distinguish between two problems which satisfy the above hypotheses and differ only in the end-conditions $\Psi_{\gamma}{ }^{1}=0$ and $\Psi_{\gamma}{ }^{2}=0,(\gamma=1, \cdots, p)$, respectively, and these conditions are such that the matrix

$$
\begin{aligned}
& \left|\begin{array}{cc}
\Psi_{\gamma ; j 1}^{1} & \Psi_{\gamma ; j 2}^{1} \\
\Psi_{\gamma ; j 1}^{2} & \Psi_{\gamma ; j 2}^{2} \\
-v_{j h}\left(x_{1}\right) & v_{j h}\left(x_{2}\right)
\end{array}\right|, \\
& (j=1, \cdots, n ; \gamma=1, \cdots, p ; h=1, \cdots, r),
\end{aligned}
$$

has rank $p+r$. If a problem satisfies $\left(\mathrm{H}_{1}\right)$ and $\left(\mathrm{H}_{2}\right)$, and not $\left(\mathrm{H}_{3}\right)$, then the matrix (23) has rank $p+r-k$, where $0<k \leqq p$. By deleting $k$ of the end-conditions $\Psi_{\gamma}=0,(\gamma=1, \cdots, p)$, one may then obtain a problem which satisfies $\left(\mathrm{H}_{3}\right)$, and which is equivalent to the original problem in the sense that an $\operatorname{arc} \eta$ is admissible for this new problem if and only if it is admissible for the original problem. Such a modified problem will be called the normal boundary problem determined by the end-conditions $\Psi_{\gamma}=0,(\gamma=1, \cdots, p)$.

3. Existence of Characteristic Values. As indicated in the introduction, diverse methods of proof have been used in treating various special boundary problems of the general type formulated in the preceding section. This is particularly true of such boundary problems that are linear in the characteristic parameter. In this section we shall discuss the different methods that have been utilized in the proof of the existence of characteristic values. Instead of attempting to discuss the papers chronologically we shall group them according to the methods employed. Moreover, we shall discuss first those methods which have been used less within recent years in the consideration of boundary problems associated with the calculus of variations.

(a) Application of the theory of integral equations. As shown by Hilbert [1], a boundary value problem consisting of a single second order differential equation with real coefficients and corresponding self-adjoint boundary conditions is equivalent to an integral equation with a real symmetric closed kernel. Hence by Hilbert's theory of such integral equations the given boundary problem has an infinite number of real and no complex char- 
acteristic values. The results of the papers of Cairns, Mason [1], and Myller [1] listed in the bibliography either depend directly upon, or are closely related to the Hilbert theory of integral equations. Marković ([1] and [2]) has recently applied the theory of integral equations to the study of periodic solutions of selfadjoint equations of even order with periodic coefficients. $\dagger$

By the introduction of a Green's matrix the general boundary problem (17), (18) is reduced to the equivalent system of integral equations

$$
\eta_{i}(x)=\lambda \int_{x_{1}}^{x_{2}} \kappa_{i j}(x, t) \eta_{j}(t) d t,
$$

where $\kappa_{i j}(x, t) \equiv \kappa_{j i}(t, x)$. The relation of the linear system of the form (17), (18) to the more general system (21) will be discussed later.

(b) Application of difference equations. The method of difference equations has served as a heuristic approach to many kinds of transcendental problems. Indeed, it was the unpublished method which Sturm originally used in his investigations of boundary value problems. The rigorous passage to the limit from a system of difference equations to a differential system was carried through by Porter in 1902. Since then this method has been used by Bôcher, Fort, Carmichael, Richardson, Courant, Plancherel, W. M. Whyburn, and others in considering various types of boundary problems. We may, therefore, think of this method of passage to the limit as one of the well-established methods of treating such problems. If the speaker may venture a personal opinion, however, it seems that most results which have been established by the use of difference equations have subsequently been proved by other methods more elegant in detail.

(c) Application of asymptotic expressions. The use of asymptotic expressions for the characteristic values and corresponding characteristic functions, together with the use of these expressions in the theory of the development of arbitrary functions, dates from the work of Liouville. The reader is referred to

$\dagger$ For brevity, references to literature on special equations, such as those of Hill and Mathieu, are not included in the bibliography at the end of this paper. 
Bôcher [1], pp. 177-179, for an introductory discussion of this method for a boundary problem consisting of a single second order differential equation and Sturmian boundary conditions. In more recent times, Birkhoff [1] has developed asymptotic expressions for the solutions of a single equation of the $n$th order involving a parameter in a general fashion, and has applied his results to a boundary problem which is linear in the characteristic parameter, and involves two-point boundary conditions. The coefficients of the differential equation and the boundary conditions of his problem are not assumed real; moreover, the system itself is not supposed to be self-adjoint. In certain general cases Birkhoff obtains the existence of infinitely many characteristic values, and a corresponding expansion theorem for arbitrary functions. In view of Birkhoff's general assumptions, it is not surprising that those real self-adjoint systems which come under the class considered by him may be treated by other methods which are simpler in detail than that involving the use of asymptotic expressions. All of the problems associated with the second variation of calculus of variations problems in the manner described in the preceding section are real and selfadjoint. Consequently we shall not consider in detail the results of Birkhoff, nor shall we discuss specifically the important subsequent work of Birkhoff, Tamarkin, Langer, Stone, and others in this field. For completeness, their papers are listed in the bibliography at the end of this paper. It is to be remarked in passing, however, that in the field of convergence and equiconvergence theorems no other method has as yet obtained as general theorems as those proved by the use of asymptotic expressions.

(d) Application of linear algebraic equations in infinitely many variables. In 1914 Lichtenstein [1] considered boundary value problems involving a single second order linear differential equation linear in the characteristic parameter, with associated Sturmian boundary conditions. By expanding admissible functions $\eta(x)$ in Fourier series, he showed that the characteristic values of the given problem were identical with the characteristic values of an infinite system of linear algebraic equations

$$
\left(\delta_{i j}+\lambda \kappa_{i j}\right) x_{j}=0, \quad(i, j=1,2, \cdots),
$$

in the variables $\left(x_{i}\right)$. The matrix $\left\|\kappa_{i j}\right\|$ is completely continuous 
(vollstetig) in the sense of Hilbert. Under certain general conditions he proved the existence of infinitely many characteristic values for the given problem, and also established equi-convergence theorems in terms of the characteristic functions. Later, Lichtenstein [2] extended the method of proof to a system consisting of a second order integro-differential equation with the special boundary conditions $\eta\left(x_{1}\right)=0=\eta\left(x_{2}\right)$. Geiringer [1] has generalized the Lichtenstein method of proof to a system of differential equations of the second order, and Boerner [1] has used the method in considering a self-adjoint equation of the fourth order. Anna Pell Wheeler has applied the theory of linear algebraic equations in an infinite number of variables to a second-order differential equation with Sturmian boundary conditions. Her method is very intimately related to that of Lichtenstein, though not identical with it. Finally, E. Hölder has extended the Lichtenstein method to the boundary problem associated with the second variation of the problem of Lagrange with fixed end points, that is, to a system (17) with the boundary conditions $\eta_{i}\left(x_{1}\right)=0=\eta_{i}\left(x_{2}\right)$.

(e) Application of general theorems on differential equations. Bliss [2] considered a boundary problem of the form

$$
\begin{array}{lr}
y_{\sigma}^{\prime}=\left[\mathfrak{A}_{\sigma \tau}(x)+\lambda \mathfrak{B}_{\sigma \tau}(x)\right] y_{\tau}, & x_{1} \leqq x \leqq x_{2}, \\
\mathfrak{M}_{\sigma \tau} y_{\tau}\left(x_{1}\right)+\mathfrak{N}_{\sigma \tau} y_{\tau}\left(x_{2}\right)=0, & (\sigma, \tau=1, \cdots, \mathfrak{n}),
\end{array}
$$

where the functions $\mathfrak{H}_{\sigma \tau}, \mathfrak{B}_{\sigma \tau}$ are real-valued and continuous on $x_{1} x_{2}$, and $\mathfrak{M}_{\sigma \tau}, \mathfrak{N}_{\sigma \tau}$ are real constants with the matrix $\left\|\mathfrak{M}_{\sigma \tau} ; \mathfrak{R}_{\sigma \tau}\right\|$ of rank $\mathfrak{n}$. The problem adjoint to (25) is defined as

$$
\begin{gathered}
z_{\sigma}^{\prime}=-z_{\tau}\left[\mathfrak{U}_{\tau \sigma}(x)+\lambda \mathfrak{B}_{\tau \sigma}(x)\right], \\
z_{\tau}\left(x_{1}\right) \mathfrak{P}_{\tau \sigma}+z_{\tau}\left(x_{2}\right) \mathfrak{\bigcap}_{\tau \sigma}=0,
\end{gathered}
$$

where the coefficients $\mathfrak{P}_{\tau \sigma}, \mathfrak{\bigcap}_{\tau \sigma}$ satisfy the relations

$$
\mathfrak{M}_{\sigma \nu} \mathfrak{B}_{\nu \tau}-\mathfrak{R}_{\sigma \nu} \mathfrak{Q}_{\nu \tau}=0, \quad(\sigma, \nu, \tau=1, \cdots, \mathfrak{n}) .
$$

This definition of adjoint system is equivalent to that originally introduced by Bounitzky [1]. Bliss called the system (25) selfadjoint if it is equivalent to (26) under a non-singular linear transformation $z_{\sigma}=\mathfrak{I}_{\sigma \tau}(x) y_{\tau}$. When a further hypothesis was satisfied the system was termed definitely self-adjoint. Bliss proved that a definitely self-adjoint system has only real char- 
acteristic values, and that these values are denumerably infinite in number. Moreover, certain general expansion theorems were proved by methods analogous to those of the Hilbert-Schmidt integral equation theory.

The system (17), (18) is self-adjoint with respect to the transformation matrix

$$
\|\mathfrak{I}\| \equiv\left\|\begin{array}{rr}
0_{i j} & \delta_{i j} \\
-\delta_{i j} & 0_{i j}
\end{array}\right\|, \quad \quad(i, j=1, \cdots, n) .
$$

This system, however, is definitely self-adjoint according to the definition of Bliss [2] only in case $\left|B_{i j}\right| \neq 0$, that is, in case there are no auxiliary differential equations $\Phi_{\alpha}=0$. In this connection it is to be pointed out that Cope [1] makes the erroneous statement that the general system (17), (18) is definitely self-adjoint according to the definition of Bliss [2], and this incorrect statement has not been eliminated in the slightly changed form of his thesis which has only recently been published.

$\mathrm{Hu}$ [1] has considered specifically the system (17), (18), and by a method of proof similar to that of Bliss [2] has proved the existence of an infinity of characteristic values. Moreover, the existence theorem of $\mathrm{Hu}$ does not assume the strengthened Clebsch condition $\left(\mathrm{H}_{2}\right)$, but simply the non-singularity of the matrix (13). In a recent paper Bliss [5] has modified the definition of definite self-adjointness in such a way that the system (17), (18) is definitely self-adjoint according to this new definition. However, a general system (25) may be definitely selfadjoint according to this new definition and have no characteristic values or only a finite number. Reid [6] has shown that a system which is definitely self-adjoint according to this new definition, and which satisfies the additional restriction that the matrix $\left\|\mathfrak{B}_{\sigma \tau}\right\|$ is of constant rank on $x_{1} x_{2}$, is equivalent to a system of the form (22). The character of the equivalence of the given system to this latter system is new in the sense that the second system involves twice the number of dependent functions occurring in the original system. Conditions are given by Reid [6] for such a system to have an infinity of characteristic values. In particular, the problem considered by $\mathrm{Hu}$ is such a definitely self-adjoint system.

(f) Application of the minimum principle. Now consider a boundary problem (22) which satisfies hypotheses $\left(\mathrm{H}_{1}\right),\left(\mathrm{H}_{3}\right)$, 
for which the matrix (13) is non-singular, and such that the corresponding expression $J_{2}[\eta]$ is positive for all non-identically vanishing admissible arcs $\eta$. These conditions imply that the strengthened Clebsch condition $\left(\mathrm{H}_{2}\right)$ also holds. It is to be noted that whenever the form $\eta_{i} K_{i j} \eta_{j}$ is positive definite and $\left(\mathrm{H}_{2}\right)$ is satisfied, the hypothesis $J_{2}[\eta]>0$ for non-identically vanishing admissible arcs is not an essential restriction. In this case a value $\lambda_{0}$ exists such that if $J_{2}[\eta]$ is replaced by $J_{2}[\eta]$ $+\lambda_{0} \int_{x}^{x_{2}} \eta_{i} K_{i j} \eta_{j} d x$ the modified quadratic functional satisfies the desired condition. The modified boundary problem is equivalent to the original problem by a linear change of parameter.

There will now be defined a sequence of classes of admissible arcs in which we shall consider the problem of minimizing the functional $J_{2}[\eta]$. The class $S_{1}$ is defined as the totality of admissible arcs satisfying the relation

$$
\int_{x_{1}}^{x_{2}} \eta_{i} K_{i j} \eta_{j} d x=1
$$

The sequence of classes is defined by induction as follows: suppose classes $S_{1}, \cdots, S_{s-1},(s \geqq 2)$, have been defined and are not empty, and for $\lambda=\lambda_{t},(t=1, \cdots, s-1)$, where $\lambda_{t}$ is the greatest lower bound of $J_{2}[\eta]$ in the class $S_{t}$, there are $r_{t},\left(0<r_{t} \leqq 2 n\right)$, linearly independent solutions of (22). If $\eta_{i \kappa}, \zeta_{i k},(\kappa=1,2, \cdots, r$ $\left.=r_{1}+\cdots+r_{s-1}\right)$, denote these characteristic solutions, the class $S_{s}$ is defined as the totality of arcs $\eta$ of the class $S_{s-1}$ which satisfy the relations

$$
\int_{x_{1}}^{x_{2}} \eta_{i \kappa} K_{i j} \eta_{j} d x=0, \quad(\kappa=1, \cdots, r) .
$$

Under the above hypotheses one may then prove the following theorem: If the class $S_{s}$ is non-vacuous and $\lambda_{s}$ is the greatest lower bound of $J_{2}[\eta]$ in this class, then $\lambda=\lambda_{s}$ is a characteristic value of (22) and $\lambda_{s}>\lambda_{s-1}$.

Various writers have proved for more or less general problems and by diverse methods the above theorem in the process of showing the existence of characteristic values. For the case of a single differential equation of the second order with self-adjoint boundary conditions Mason has used this method to prove the existence of characteristic values. The first proof of Mason [1] 
depended upon the theory of integral equations, but in later papers (Mason [2], [3], and [4]) this proof was made independent of integral equation theory. A somewhat different proof of this result for a second order differential equation with Sturmian boundary conditions was given by Holmgren [1]. Reid [1] proved the above theorem for the general problem (22) by a method which closely parallels that of Mason.

Still more recently Reid [5], using methods analogous to those of Reid [1], has treated a type of integro-differential boundary problem which contains the differential boundary problems formulated in $\$ 2$ as special cases. In Reid [5] there is first considered a self-adjoint integro-differential boundary problem related to the functional $J_{2}[\eta]+\int_{x_{1}}^{x_{2}} \int_{x_{1}}^{x_{2}} M_{i j}(x, t) \eta_{i}(x) \eta_{j}(t) d x d t$ in the manner that the problem (17), (18) is related to $J_{2}[\eta]$. Such a system reduces to (17), (18) for $M_{i j}(x, t) \equiv 0$. Under the assumption that the expressions $J_{2}[\eta], \Phi_{\alpha}\left(x, \eta, \eta^{\prime}\right), \Psi_{\gamma}\left[\eta\left(x_{1}\right), \eta\left(x_{2}\right)\right]$ satisfy hypotheses $\left(\mathrm{H}_{1}\right),\left(\mathrm{H}_{2}\right),\left(\mathrm{H}_{3}\right)$, while the functions $M_{i j}(x, t)$ are continuous and $M_{i j}(x, t)=M_{j i}(t, x)$, it is proved that this accessory integro-differential problem has infinitely many characteristic values. There is also considered in Reid [5] an integrodifferential problem that in general involves the parameter $\lambda$ non-linearly, and which reduces to the differential system (21) when certain functions are identically zero. The method of treatment is that of considering an auxiliary boundary problem which is linear in a second characteristic parameter $\Lambda$ and related to the quadratic functional $J_{2}[\eta: \lambda]$ of the given problem in a manner analogous to that in which the differential system (21) is related to (20). The characteristic values of the given problem are then those values of $\lambda$ for which some characteristic value of the auxiliary problem is equal to zero. Several sets of sufficient conditions for the existence of infinitely many characteristic values are given, and, when stated for the special problems of the form (21) previously considered by Morse and Birkhoff and Hestenes, these results go beyond those obtained by the authors just mentioned.

For the accessory boundary problem (17), (18), that is, the system (22) with $K_{i j}=\delta_{i j}$, the above theorem may be established readily by the use of theorems of the classical calculus of variations. This was first indicated in a short paper by Bliss [1] for a plane problem of the calculus of variations with variable 
end points. He proved by calculus of variations methods the extremizing property of the smallest characteristic value of the associated boundary problem, and established the equivalence of the non-negativeness of the characteristic values and the usual Jacobi condition. Hickson [1] treated the accessory boundary problem for a fixed end-point problem of the calculus of variations in $n$-space by the use of classical theorems of the calculus of variations. Finally, this calculus of variations method has been extended to the general problem (17), (18) by Wiggin [1]. One might also establish the above theorem for the problem (22) by similar methods, but in view of the results of Reid [5], \$7, one may readily deduce this result from the corresponding theorem for the accessory problem (17), (18).

Another type of proof of the above theorem is given by the existence theorems for the problem of Mayer that have been established by Graves [1].

One may also define the characteristic values by means of the maximum-minimum property introduced by Courant, which does not involve the use of characteristic functions. This method is particularly useful in the treatment of partial differential and more general functional equations.

For a discussion of the application of the method of Ritz to certain self-adjoint boundary problems the reader is referred to Kryloff [1]. This memoir contains a bibliography of the numerous publications of Kryloff and others on this topic.

(g) Application of comparison and oscillation theorems. As indicated in the introduction, Sturm utilized comparison and oscillation theorems in the proof of the existence of infinitely many characteristic values for the problem he considered. Bôcher greatly simplified the details and extended the results of the Sturmian theory. For a bibliography of the extensive publications of Bôcher the reader is referred to Birkhoff [4]. For brevity, Bôcher's papers are not listed separately in the bibliography of the present paper. Further results on boundary problems associated with a single second order differential equation have been proved by Ettlinger ([1] and [2]).

In his statement of sufficient conditions for the problem of Bolza, Morse [2] used the boundary value problem formulation of the Jacobi condition, established for this problem comparison and oscillation theorems, and showed the existence of infinitely 
many characteristic values. In his Colloquium lecture Morse [3] developed more general separation, comparison, and oscillation theorems for problems which are in general non-linear in the parameter. Although his results are stated for the problem in $n$-space without auxiliary differential equations, the methods are seen to extend readily to the general problem of Bolza when suitable normality assumptions are made. From the standpoint of the existence of characteristic values we may say that Morse's method is that of comparison and oscillation theorems, since such theorems are first established and then used in the proof of the existence of infinitely many characteristic values. The comparison and oscillation theorems, together with his results on the existence of characteristic values, are obtained under hypotheses which are entirely analogous to those of the Sturmian theory. In particular, it is assumed that for arbitrary non-identically vanishing admissible arcs $\eta$ the functional (20) is a proper monotone decreasing function of $\lambda$.

Birkhoff and Hestenes have also treated boundary value problems associated with problems of the calculus of variations involving no auxiliary differential equations. Their results on the existence of characteristic values, similar to those of Morse, are also obtained by the use of comparison and oscillation theorems. Further discussion of their results will be given in the next section on comparison and oscillation theorems.

4. Oscillation and Comparison Theorems. In the introduction we have indicated the general character of the comparison and oscillation theorems of the Sturmian theory. The present section is concerned with the great extensions and generalizations of these theorems that have been established within recent years. Before taking up this more recent literature, however, we note that for a second order equation linear in the parameter and having associated Sturmian boundary conditions Richardson [1] proved the oscillation properties of the characteristic functions using the Jacobi condition of the calculus of variations and the extremizing properties of the characteristic values. This last property was deduced from integral equation theory.

As indicated in $\$ 3(\mathrm{~b})$, various writers have reconstructed and extended Sturm's work on second-order difference equations, proving oscillation and comparison theorems for such equations. Results for differential systems have been obtained by limiting 
processes from corresponding results for difference equations by Porter, Bôcher, Whyburn, and others. The papers [2] and [3] of Carmichael contain a highly interesting treatment of difference equations, together with a discussion of the intimate relationships that exist between algebraic and transcendental problems.

The boundary problem for which Morse ([1] and [3]) states his general comparison and oscillation theorems is equivalent to a problem (21) which does not involve any auxiliary differential equations $\Phi_{\alpha}=0$. It is assumed that for each value of $\lambda$ hypothesis $\left(\mathrm{H}_{2}\right)$ is satisfied, and for $\lambda$ sufficiently small the functional (20) is positive for arbitrary non-identically vanishing admissible arcs $\eta$; moreover, conditions are imposed which insure that for non-identically vanishing admissible arcs $\eta$ the functional $J_{2}[\eta: \lambda]$ is a properly monotone decreasing function of $\lambda$. For such a problem $\left(\mathrm{H}_{3}\right)$ is satisfied whenever the matrix $\left\|\Psi_{\gamma ; i 1} \Psi_{\gamma ; i 2}\right\|$ is of rank $p$. By the use of broken extremals, Morse introduces a quadratic form in a finite number of variables whose coefficients depend upon $\lambda$ and which has the following property: the form is singular if and only if $\lambda$ is a characteristic value of (21); moreover, for such a value the nullity of the form is the index of $\lambda$ as a characteristic value, and the negative type number of the form is equal to the number of characteristic values of (21) which are less than this value.

Suppose that $B$ is the problem determined by $Q, \omega, \Psi_{\gamma}$, $(\gamma=1, \cdots, p), B^{*}$ is a second problem determined by $Q^{*}, \omega^{*}$, $\Psi_{\nu}^{*},\left(\nu=1, \cdots, p^{*}\right)$, and that each of these problems satisfies the above described conditions. We shall denote the characteristic values of $B$ and $B^{*}$ by $\left\{\lambda_{s}\right\}$ and $\left\{\lambda_{s}^{*}\right\},(s=1,2, \cdots)$, respectively, whenever these values exist. If the relations $\Psi_{\gamma}\left[\eta\left(x_{1}\right), \eta\left(x_{2}\right)\right]$ are linearly dependent on those of the set $\Psi_{\nu}^{*}\left[\eta\left(x_{1}\right), \eta\left(x_{2}\right)\right]$, while $Q \equiv Q^{*}, \omega \equiv \omega^{*}$, Morse has called $B^{*}$ a sub-problem of $B$ of dimension $p^{*}-p$.

In case $B$ and $B^{*}$ have $\omega \equiv \omega^{*}$ Morse has proved comparison theorems of the following nature: $(\alpha)$ comparison of a problem with a sub-problem; $(\beta)$ comparison of two problems which differ only in the end form $Q ;(\gamma)$ comparison of two general problems.

The essential result of type $(\alpha)$ may be stated as follows: Suppose $B^{*}$ is a sub-problem of $B$ of dimension $p^{*}-p$. Then if $\lambda_{1}, \cdots, \lambda_{s+p^{*}-p}$ exist for $B$ the characteristic values $\lambda_{1}{ }^{*}, \cdots, \lambda_{s}{ }^{*}$ exist for $B^{*}$ and $\lambda_{s+p^{*}-p} \geqq \lambda_{s}^{*} \geqq \lambda_{s},(s=1,2, \cdots)$. 
In case $(\beta)$ let $N$ and $P$ denote, respectively, the negative and positive type numbers of the quadratic form $Q^{*}-Q$ on the linear set of values $\left[\eta_{i}\left(x_{1}\right), \eta_{i}\left(x_{2}\right)\right]$ satisfying $\Psi_{\gamma}=0$. Then if $\lambda_{1}, \cdots, \lambda_{s+P}$ exist for $B$, the characteristic values $\lambda_{1}^{*}, \cdots, \lambda_{s}^{*}$ exist for $B^{*}$ and $\lambda_{s+P} \geqq \lambda_{s}^{*} ;$ if $\lambda_{1}{ }^{*}, \cdots, \lambda_{s+N}^{*}$ exist for $B^{*}$, then $\lambda_{1}, \cdots, \lambda_{s}$ exist for $B$ and $\lambda_{s+N}^{*} \geqq \lambda_{s}$.

In the general case $(\gamma)$ let $\Psi_{\rho}^{0}=0,\left(\rho=1, \cdots, p^{0}\right)$, be linearly independent conditions such that the problem $B_{0}$ involving $Q, \omega$, $\Psi_{\rho}^{0}$ is a sub-problem of $B$, and the problem $B_{0}^{*}$ involving $Q^{*}, \omega, \Psi_{\rho}^{0}$ is a sub-problem of $B^{*}$. The results of Morse in case $(\gamma)$ are obtained by applying the results of the previous cases to the problems $B$ and $B_{0}, B^{*}$ and $B_{0}^{*}, B_{0}$ and $B_{0}^{*}$. In particular, if $B$ and $B^{*}$ are two general problems the number of characteristic values of $B$ on a given finite interval of the $\lambda$-axis differs from the number of characteristic values of $B^{*}$ on this interval by at most $2 n$.

For the case of different integrand functions $\omega$ we shall state only the following result: If $J_{2}^{*}[\eta: \lambda]$ and $J_{2}[\eta: \lambda]$ denote the expressions (20) for $B^{*}$ and $B$, respectively, and $J_{2}^{*}[\eta: \lambda] \geqq J_{2}[\eta: \lambda]$ for arbitrary non-identically vanishing admissible arcs $\eta$, then if $\lambda_{1}^{*}, \cdots, \lambda_{s}^{*}$ exist for $B^{*}$ the characteristic values $\lambda_{1}, \cdots, \lambda_{s}$ exist for $B$ and $\lambda_{s}^{*} \geqq \lambda_{s}$. If the strict inequality sign holds in the first relation, then the strict inequality sign also holds in the second relation.

The general oscillation theorem of Morse [3] is as follows: If $v(\lambda)$ is the number of characteristic values of $B$ less than $\lambda$, and $c(\lambda)$ is the number of points on $x_{1}<x<x_{2}$ conjugate to $x_{1}$ for this value of $\lambda$, then $v(\lambda)-(2 n-p) \leqq c(\lambda) \leqq v(\lambda)$.

Birkhoff and Hestenes have also obtained comparison and oscillation theorems for the same problem that has been treated by Morse [3]. By the introduction of so called natural isoperimetric conditions for the functional $J_{2}[\eta]$, they are led to a characterization of the solutions of the corresponding equations $J_{i}(\eta)=0$ which satisfy the boundary conditions (17). In particular, a set of natural isoperimetric conditions is called a minimal set if $J_{2}[\eta] \geqq 0$ for every admissible arc $\eta$ satisfying these conditions, and if no proper subset of these conditions has this property. The number of conditions in a minimal set is always the same, and this number is called the type number of $J_{2}[\eta]$ relative to the conditions $\Psi_{\gamma}=0$. For a non-singular problem this 
type number is finite if and only if hypothesis $\left(\mathrm{H}_{2}\right)$ holds. In addition, Birkhoff and Hestenes introduce the term order of degeneracy for the maximum number of linearly independent solutions of $J_{i}[\eta]=0$ satisfying the boundary conditions (18). If $\left(\mathrm{H}_{2}\right)$ is satisfied, the type number and order of degeneracy of $J_{2}[\eta]$ relative to $\Psi_{\gamma}=0$ are equal respectively to the negative type number and the nullity of the Morse quadratic form. The oscillation and comparison theorems of Birkhoff and Hestenes are somewhat more complete than those of Morse, because of the introduction of the notion of order of concavity of a problem $B$ with respect to a sub-problem $B^{*}$. Hestenes [3] has recently announced the extension of these results to the general boundary problem associated with the problem of Bolza as formulated in $\$ 2$.

Because of their generality, we have first described the comparison and oscillation theorems obtained by Morse and Birkhoff and Hestenes for the general problem (21). It is to be noted, however, that for the problem (17), (18) which involves the parameter linearly these comparison and oscillation theorems are deducible from the extremizing properties of the characteristic values. $\mathrm{Hu}[1]$ has used this method in proving certain comparison and oscillation theorems for the accessory problem. This method has also been used by Reid [5] in establishing general comparison and oscillation theorems for the integrodifferential problem there considered. Moreover, as pointed out in the preceding section, Reid has shown that the theory for the general boundary problem (21) may be made to depend upon an associated boundary problem involving a second parameter linearly. Comparison and oscillation theorems for the general problem are ready consequences of the corresponding theorems for the associated problem. This method of treating the general problem non-linear in the parameter seems to be of distinct interest because of the diverse methods of proof that are available for the treatment of the associated problem linear in the second parameter. It is to be noted that in order to establish an oscillation theorem for the general problem (21) when no additional assumption of normality is made it is necessary to formulate a new definition of conjugate points. The definition given by Reid [5] for the integro-differential problem there treated reduces for the differential problem (21) to the follow- 
ing: For $x_{1}<c<x_{2}$ let $B_{c}$ denote the normal boundary problem determined by the functional $J_{2}[\eta: \lambda]$ and the end-conditions $\eta_{i}\left(x_{1}\right)=0=\eta_{i}(c)$. The value $c$ is said to be a conjugate point of $x=x_{1}$ on $x_{1} x_{2}$ relative to the differential equations of (21) for $\lambda=\lambda_{0}$ if: (i) $\lambda=\lambda_{0}$ is a characteristic value of $B_{c}$; (ii) there is at least one characteristic solution $\eta_{i}=y_{i}, \zeta_{i}=z_{i}$ of $B_{c}$ such that for arbitrary $\epsilon$ satisfying $0<\epsilon<x_{2}-c$ there is no corresponding set of functions $\zeta_{i}(x)$ forming with the arc $\eta_{i}=y_{i}(x)$ on $x_{1} c, \eta_{i} \equiv 0$ on $c \leqq x \leqq c+\epsilon$ a characteristic solution of $B_{c+\epsilon}$ for $\lambda=\lambda_{0}$. It is easily seen that this definition of a conjugate point reduces to the older definition when certain additional assumptions of normality are satisfied.

5. Application of Boundary Value Problems to Sufficiency Theorems in the Calculus of Variations. As indicated in the introduction for a simple problem of the calculus of variations, the Jacobi condition may be phrased in terms of a boundary value problem. For a detailed discussion of this approach for a simple problem the reader is referred to chapter VI of Lovitt [1]. Lichtenstein [3] and [4] has used the method of boundary problems in obtaining sufficient conditions for a weak relative minimum in certain problems of the calculus of variations. Boerner [2] and [3] has applied the method of Lichtenstein to problems involving higher derivatives and to the parametric problem in the plane with fixed end-points. As indicated in $\S 3(\mathrm{f})$, Bliss [1] has expressed the Jacobi condition for a plane calculus of variations problem with variable end-points in terms of the characteristic values of a boundary problem. For the use of this formulation of the Jacobi condition in connection with sufficiency theorems for more general problems of the calculus of variations, the reader is referred to Morse [2], Myers [1], Hestenes [1] and [2], Bliss [4], and Reid [3] and [4]. A comparison of different formulations of the Jacobi condition has been made by Reid [2]. It is of interest to note that the boundary problem used by Reid [3] in treating discontinuous solutions in the non-parametric problem involves boundary conditions at more than two points. By a very simple transformation, however, this problem is reducible to one of the form (17), (18) with two-point boundary conditions.

6. Remarks. We shall now discuss briefly certain aspects of 
the theory of boundary problems of the type formulated in $\$ 2$ that are in need of further consideration. It follows readily that a real self-adjoint boundary problem consisting of a single differential equation of even order and associated two-point boundary conditions may be reduced to a problem of the sort formulated in \$2. Thus, for example, all the theorems of Janczewsky [1] and Cimmino [1] on the existence of characteristic values for a real self-adjoint fourth order boundary problem follow readily from the theory of the general problem of this paper. However, from the oscillation theorems that have been established for this general problem one does not obtain all of the oscillation theorems that have been proved for real self-adjoint boundary problems whose differential equations are of even order greater than two. As yet no one has made an exhaustive study of the relations between comparison and oscillation theorems for such a system and the corresponding theorems for the general problem of $\$ 2$.

In all of the above discussion of the general problems of $\$ 2$ we have assumed that the matrix (13) is non-singular. We do not have time in this lecture to consider the work of Hilbert, Weyl, and others concerning singular boundary value problems. We wish to mention, however, a recent paper by Morse and Leighton on singular quadratic functions because their method of attack is that of the calculus of variations. It would be highly desirable to extend their work to singular quadratic functionals involving more than a single dependent function, and to consider the theory of associated boundary value problems.

As indicated in $\$ 3(\mathrm{c})$, various authors have obtained by the use of asymptotic expansions stronger results for the expansion problem than have been obtained by other methods. The question of the convergence and summability properties of the generalized Fourier expansions in terms of the characteristic functions of the general boundary problem here considered is a fertile field for further research.

There is also the question of boundary problems of the type formulated in $\$ 2$ which involve more than a single characteristic parameter. Hilbert [1], Yoshikawa [1], [2], Hilb [1], Richardson [2], [3], and [4], and others have considered the question of a Klein oscillation theorem for boundary problems associated with second order differential equations. To date this 
question has not been considered in detail for more general problems of the type here discussed. A start in this direction has been made by Reid [7].

Another field of interest is the consideration of the character of solutions of the boundary problem when the independent variable $x$ is complex. Hille has considered rather extensively the question of oscillation theorems for second order differential equations in the complex domain. So far as I know, however, such a study has not been made of more general problems of the sort considered in this paper.

Finally, the question of the use of boundary problems in considering more general functionals of the calculus of variations is of distinct importance. In his recent Chicago dissertation Goldstine [1] has considered a somewhat general functional of lines, and has formulated an analogue of the Jacobi condition in terms of an integral equation. It is of interest to note that for the classical calculus of variations problem his formulation of the Jacobi condition is expressible in terms of a boundary problem of the fourth order.

7. Boundary Problems Associated with Multiple Integrals of the Calculus of Variations. In concluding this address, I wish to speak very briefly concerning boundary value problems associated with double and multiple integrals of the calculus of variations. For a double integral problem involving a single dependent function the Jacobi equation along an extremal which satisfies the strengthened Legendre condition is an elliptic partial differential equation of the second order. Consequently all the literature concerning such partial differential equations may be thought of as concerning the Jacobi equation for a calculus of variations problem. We shall, however, restrict our comments here to the discussion of a few papers which are specifically concerned with the phrasing of the Jacobi condition for double and multiple integrals in terms of a boundary problem associated with the Jacobi equation.

Lichtenstein [7], [8], and [9] has utilized such a formulation of the Jacobi condition for double integral problems with fixed boundary and also for such problems involving variable boundary conditions of a very simple type. He assumed that the integrand function was analytic, and by the use of expansion theorems in terms of the characteristic functions of the bound- 
ary problem he proved that when the smallest characteristic number of his problem was greater than unity the given extremal surface afforded the integral a weak relative minimum Picone [2] has also expressed the Jacobi condition in terms of the characteristic values of a boundary problem. Reid [8] established by use of methods of the calculus of variations the minimizing property of the least characteristic number of the associated boundary problem, and obtained a corresponding proof of sufficient conditions for a weak relative minimum for the double integral problem with fixed boundary values. Reid [9] has given an extended form of the Jacobi condition which is of use in considering the associated boundary value problem when the integrand function and the given extremal are not assumed to be analytic.

For double and $n$-tuple integrals of the calculus of variations involving a single dependent function Simmons ([1] and [2]) has formulated an analogue of the Jacobi condition in terms of a boundary problem. One may readily formulate such an analogue for still more general multiple integrals of the calculus of variations.

Using the theory of boundary value problems, Birkhoff and Hestenes have proved the existence of a set of natural isoperimetric conditions for the second variation along an extremal of a double integral problem which satisfies the strengthened Legendre condition.

It is to be remarked that for these accessory problems associated with double and multiple integral problems one may prove certain comparison theorems; however, the results which have been obtained concerning oscillation theorems for such problems are quite meager.

The properties of minimizing surfaces for double and multiple integral problems which are not extremals in the usual sense, that is, which are not of class $C^{2}$, have been studied by Haar and his associates (see Haar [1]; also Bliss [7]). For such a minimizing surface the usual Jacobi equation is replaced by a pair of partial differential equations. One may readily set up a corresponding boundary problem and state that as a necessary condition for a minimum this problem can have no negative characteristic values. However, the question of the existence and the character of the solutions of such a boundary value 
problem has not yet been solved. The study of this sort of boundary problem and its application to the proof of sufficient conditions for an extremum in such a problem is one of distinct interest and importance in the calculus of variations.

\section{BIBLIOGRAPHY}

The following bibliography on boundary value problems associated with simple integrals of the calculus of variations includes not only the papers referred to in this address, but also many related papers published since 1900 . Although extensive, it is doubtless incomplete. The part of this bibliography related to multiple integral problems of the calculus of variations is much less pretentious, containing only papers to which explicit reference is made. The reader is referred to the encyclopedic article of Lichtenstein [Encyclopädie der Mathematischen Wissenschaften, II C, 12, pp. 1276-1334] for references to literature prior to 1924 on second order partial differential equations of elliptic type. Because of their frequent occurrence the following abbreviations are used: American Journal of Mathematics: A.J.M.; Annals of Mathematics: Ann. M.; Mathematische Annalen: M.A.; Mathematische Zeitschrift: M.Z.; Transactions of the American Mathematical Society: T.A.M.S.

\section{BOUNDARY PROBLEMS ASSOCIATED WITH SIMPLE INTEGRALS OF THE CALCULUS OF VARIATIONS}

BAMFORTH, F. R.

1. A classification of boundary problems for a system of ordinary differential equations containing a parameter, Dissertation, Chicago, 1928.

BIRKHOFF, G. D.

1. On the asymptotic character of the solutions of certain linear differential equations containing a parameter, T.A.M.S., vol. 9 (1908), pp. 219-231.

2. Boundary value and expansion problems of ordinary linear differential equations, T.A.M.S., vol. 9 (1908), pp. 373-395.

3. Existence and oscillation theorems for a certain boundary value problem, T.A.M.S., vol. 10 (1909), pp. 259-270.

4. The scientific work of Maxime Bôcher, this Bulletin, vol. 25 (1919), pp. 197-215.

5. (With LANGER, R. E.). The boundary problems and developments associated with a system of ordinary linear differential equations of the first order, Proceedings of the American Academy of Arts and Sciences, vol. 58 (1923), pp. 51-128.

6. (With Hestenes, M. R.). Natural isoperimetric conditions in the calculus of variations, Duke Mathematical Journal, vol. 1 (1935), pp. 198-286. 
Buiss, G. A.

1. A boundary value problem in the calculus of variations, this Bulletin, vol. 32 (1926), pp. 317-331.

2. A boundary value problem for a system of ordinary linear differential equations of the first order, T.A.M.S., vol. 28 (1926), pp. 561-584.

3. (With SchoenBerg, I. J ). On separation, comparison and oscillation theorems for self-adjoint systems of linear second order differential equations, A.J.M., vol. 53 (1931), pp. 781-800.

4. The Problem of Bolza in the Calculus of Variations, Mimeographed notes of lectures delivered winter, 1935, at the University of Chicago.

5. Definite self-adjoint boundary value problems, presented for publication to T.A.M.S.

6. The evolution of problems of the calculus of variations, American Mathematical Monthly, vol. 43 (1936), pp. 598-609.

BôchER, M.

1. Boundary problems in one dimension, Proceedings of the Fifth International Congress of Mathematicians, I, Cambridge, 1912, pp. 163-195.

2. Leçons sur les Méthodes de Sturm dans la Théorie des Équations Différentielles Linéaires, Paris, Gauthier-Villars, 1917.

(For a list of Bôcher's publications, see Birkhoff [4].) BoERnER, H.

1. Das Eigenwert problem der selbstadjungierten linearen Differentialgleichungen vierter Ordnung, M.Z., vol. 34 (1931), pp. 293-319.

2. Über einige Variationsprobleme, M.Z., vol. 35 (1932), pp. 161-189.

3. Zur Theorie der zweiten Variation, M.Z., vol. 39 (1935), pp. 492-500. BounitZKy, E.

1. Sur la fonction de Green des équations différentielles linéaires ordinaires, Journal de Mathématiques, vol. 5 (1909), pp. 65-125.

Cairns, W. D.

1. Die Anwendung der Integralgleichungen auf die zweite Variation bei isoperimetrischen Problemen, Dissertation, Göttingen, 1907.

CAMP, C. C.

1. An extension of the Sturm-Liouville expansion, A.J.M., vol. 44 (1922), pp. 25-53.

Caratheodory, C.

1. Die Theorie der zweiten Variation beim Problem von Lagrange, Münchner Berichte, 1932, pp. 99-114.

2. Über die Einteilung der Variationsprobleme von Lagrange nach Klassen, Commentarii Mathematici Helvetici, vol. 5 (1932), pp. 1-19.

Carman, M. G.

1. The expansion problem for a certain system of ordinary linear second order differential equations, A.J.M., vol. 48 (1926), pp. 169-182.

Carmichael, R. D.

1. A comparison theorem for homogeneous linear differential equations of general order, Ann. M., vol. 19 (1918), pp. 159-171.

2. Boundary value and expansion problems, A.J.M., vol. 43 (1921), pp. 69101 and 232-270, and vol. 44 (1922), pp. 129-152.

3. Algebraic guides to transcendental problems, this Bulletin, vol. 28 (1922), pp. 179-210. 
Cimmino, G.

1. Autosoluzione e autovalore nelle equazioni differenziali lineari ordinarie autoaggiunte di ordine superiore, M.Z., vol. 32 (1930), pp. 4-58.

Clemente, $P$.

1. Ricerche intorno alle soluzioni periodiche di una equazione differenziale lineare ordinaria del secondo ordine, Giornale di Matematiche di Battaglini, vol. 69 (1931), pp. 185-220.

2. Maggiorazione delle soluzioni periodiche di una equazione differenziale lineare ordinaria del secondo ordine, Atti della Reale Accademia Nazionale dei Lincei, vol. 14 (1931), pp. 89-92 and vol. 15 (1932), pp. 925-931.

Cope, $\mathrm{T}$.

1. An analogue of Jacobi's condition for the problem of Mayer with variable end points, Dissertation, Chicago, 1927; also, A.J.M., vol. 59 (1937), pp. 655672.

Courant, R.

1. (With Hilbert D.). Methoden der Mathematischen Physik, I, Berlin, 1924.

2. Über direkte Methoden bei Variations- und Randwert-problemen, Jahresbericht der Deutschen Mathematiker-Vereinigung, vol. 34 (1925), pp. 90-117.

3. Über direkte Methoden in der Variationsrechnung und über verwandte Fragen, M.A., vol. 97 (1927), pp. 711-736.

4. Über die Anwendung der Variationsrechnung in der Thecrie der Eigenschwingungen und ibber neue Klassen von Funktionalgleichungen, Acta Mathematica, vol. 49 (1927), pp. 1-68.

Davidoglou, A.

1. Etude de l'équation différentielle $\left[\theta y^{\prime \prime}\right]^{\prime \prime}=k \phi y$, Annales Scientifiques de l'École Normale Supérieure, vol. 22 (1905), pp. 539-565.

Davis, H. T.

1. An existence theorem for the characteristic numbers of a certain boundary value problem, T.A.M.S., vol. 26 (1924), pp. 1-16.

EtTLINGer, H. J.

1. Existence theorems for the general real self-adjoint linear system of the second order, T.A.M.S., vol. 19 (1918), pp. 79-96.

2. Oscillation theorems for the real self-adjoint linear system of the second order, T.A.M.S., vol. 22 (1921), pp. 136-143.

FORT, T.

1. Linear difference and differential equations, A.J.M., vol. 39 (1917), pp. 126.

2. Some theorems of comparison and oscillation, this Bulletin, vol. 24 (1918), pp. 330-334.

GEIRINGER, $\mathrm{H}$.

1. Über eine Randwertaufgabe der Theorie gewöhn icher linearer Differentialgleichungen zweiter Ordnung, M.Z., vol. 12 (1922), pp. 1-17.

Goldstine, H.

1. Conditions for a minimum of a functicnal, Dissertation, Chicago, 1936. Graves, L. M.

1. The existence of an extremum in problems of Mayer, T.A.M.S., vol. 39 (1936), pp. 456-471. 
HAAR, A.

1. Zur Theorie der orthogonalen Funktionensysteme, M.A., vol. 69 (1910), pp. 331-371, and vol. 71 (1911), pp. 38-53.

HAUPT, O.

1. Untersuchungen ilber Oszillationstheoreme, Dissertation, Würzberg, 1911.

2. Über eine Methode zum Beweise von Oszillationstheoremen, M.A., vol. 76 (1915), pp. 67-104.

3. Über ein Oszillationstheorem, Sitzungsberichte Erlangen, vol. 61 (1929), pp. 203-206.

Hestenes, M. R.

1. Sufficient conditions for the problem of Bolza in the calculus of variations, T.A.M.S., vol. 36 (1934), pp. 793-818.

2. See Birkhofr [6].

3. Quadratic functionals in the calculus of variations, abstract in this Bulletin, vol. 43 (1937), p. 205.

Hickson, A. O.

1. An application of the calculus of variations to boundary value problems, T.A.M.S., vol. 31 (1929), pp. 563-579.

HILB, E.

1. Über Kleinsche Theoreme in der Theorie der linearen Differentialgleichungen, M A., vol. 66 (1908), pp. 215-257, and vol. 68 (1910), pp. 24-74.

2. Über Reihenentwicklungen nach den Eigenfunktionen linearer Differentialgleichungen 2-ter Ordnung, M.A., vol. 71 (1911), pp. 76-87.

HILBERT, D.

1. Grundzüge einer allgemeinen Theorie der linearen Integralgleichungen, Berlin, 1912.

2. See Courant [1].

HöLDER, E.

1. Die Lichtensteinsche Methode filr die Entwicklung der zweiten Variation, angewandt auf das Problem von Lagrange, Prace Matematyczno-Fizyczne, vol. 42 (1935), pp. 307-346.

Holmaren, E.

1. Über Randwertaufgaben bei einer linearen Differentialgleichung der zweiten Ordnung, Arkiv för Matematik, Astronomi och Fysik, vol. 1 (1904), pp.401-417. Horrocks, $\mathrm{H}$.

1. Generalized Sturm-Liouville expansions in series of pairs of related functions, Proceedings of the Royal Society, London, vol. 115 (1927), pp. 184-198. Hu, K. S.

1. The problem of Bolza and its accessory boundary value problem, Contributions to the Calculus of Variations, 1931-1932, The University of Chicago Press, pp. 361-443.

Hurwitz, A.

1. An expansion theorem for a system of linear differential equations of the first order, T.A.M.S., vol. 22 (1921), pp. 526-543.

INCE, E. L.

1. Ordinary Differential Equations, London, 1927.

JACKSON, D.

1. Algebraic properties of self-adjoint systems, T.A.M.S., vol. 17 (1916), pp. 418-424. 
JACKSON, R. L.

1. The boundary value problem of the second variation for parametric problems in the calculus of variations, Dissertation, Chicago, 1928. JANCZEWSKy, S. A.

1. Oscillation theorems for the differential boundary problems of the fourth order, Ann. M., vol. 29 (1928), pp. 521-542, and vol. 31 (1930), pp. 663-680.

2. Über irreguläre Oszillationseigenschaften der Eigenfunktionen bei den Eigenwertproblemen vierter Ordnung, Comptes Rendus de l'Académie des Sciences de l'URSS, 1935I, pp. 89-92

JENSEN, M.

1. Some problems in the theory of interpolation by Sturm-Liouville functions, T.A.M.S., vol. 29 (1927), pp. 54-79.

Johnson, M. E.

1. Tensors of the calculus of variations, A.J.M., vol. 53 (1931), pp. 103-116. KLEIN, F.

1. Bemerkungen zur Theorie der linearen Differentialgleichungen zweiter Ordnung, M.A., vol. 64 (1907), pp. 175-196.

KNESER, A.

1. Untersuchungen über die Darstellung willkürlicher Funktionen in der Mathematischen Physik, M.A., vol. 58 (1904), pp. 81-147.

KöNIG, R.

1. Die Oszillationseigenschaften der Eigenfunktionen der Integralgleichungen mit definitem Kern und das Jacobische Kriterium der Variationsrechnung, Dissertation, Göttingen, 1907.

KREIN, M.

1. Sur les opérateurs différentiels oscillatoires, Comptes Rendus de l'Académie des Sciences de l'URSS, (1937), pp. 395-398.

2. Sur quelques applications des noyaux de Kellogg aux problèmes d'oscillation, Communications de la Société Mathématique de Kharkoff, vol. 11 (1935), pp. 3-19 and vol. 13 (1936), pp. 15-27.

KRYLOFF, N.

1. Les Méthodes de Solution Approchée des Problèmes de la Physique Mathématique, Mémorial des Sciences Mathématiques, fasc. 49 (1931).

LANGER, R. E.

1. See Birkhoff [5].

2. Developments associated with a boundary problem not linear in the parameter, T.A.M.S., vol. 25 (1923), pp. 155-172.

3. The boundary value problem associated with a differential equation in which the coefficient of the parameter changes sign, T.A.M.S., vol. 31 (1929), pp. 1-24.

4. The expansion problem in the theory of ordinary linear differential systems of the second order, T.A.M.S., vol. 31 (1929), pp. 868-906.

5. The boundary problem associated with a differential system rational in the parameter, T.A.M.S., vol. 32 (1930), pp. 238-250.

Latshaw, V. V.

1. The algebra of self-adjoint boundary value problems, this Bulletin, vol. 39 (1933), pp. 969-978.

LEIGHTON, W .

1. (With Morse, M.). Singular quadratic functionals, T.A.M.S., vol. 40 (1936), pp. 252-286. 
Lichtenstein, L.

1. Zur Analysis der unendlich vielen Variablen, Rendiconti del Circolo Matematico di Palermo, vol. 38 (1914), pp. 113-166.

2. Über eine Integro-Differentialgleichung und die Entwicklung willkürlicher Funktionen nach deren Eigenfunktionen, Schwarz Festschrift, Berlin, 1914, pp. 274-285.

3. Zur Variationsrechnung, I, Göttinger Nachrichten, 1919, pp. 161-192.

4. Zur Variationsrechnung, II,-Das isoperimetrische Problem, Journal für Mathematik, vol. 165 (1931), pp. 194-216.

5. Zum Sturm-Liouville Problem, M.Z., vol. 31 (1929), pp. 346-349. LovitT, W. V.

1. Linear Integral Equations, New York, 1924. MAR Ković, Ž.

1. Sur les solutions de l'équation différentielle linéaire du second ordre à coéfficient périodique, Proceedings of the London Mathematical Society, vol. 31 (1930), pp. 417-438.

2. Über die periodischen Lösungen der linearen Differentialgleichung $2 n$-ter Ordnung mit periodischen Koeffizienten, Rad jugoslavenske Akademije Znanosti i Umjetnosti, vol. 246 (1933), pp. 161-183.

MASON, M.

1. Zur Theorie der Randwertaufgaben, M.A., vol. 58 (1904), pp. 528-544.

2. On the boundary problems of linear ordinary differential equations of the second order, T.A.M.S., vol. 7 (1906), pp. 337-360.

3. The expansion of a function in terms of normal functions, T.A.M.S., vol. 8 (1907), pp. 427-432.

MerRILL, H. A.

1. On solutions of differential equations which possess an oscillation thecrem, T.A.M.S., vol. 4 (1903), pp. $423-433$.

MORSE, M.

1. A generalization of the Sturm separation and comparison theorems, M A., vol. 103 (1930), pp. 52-69.

2. Sufficient conditions in the problem of Lagrange with variable end conditions, A.J.M., vol. 53 (1931), pp. 517-546.

3. The calculus of variations in the large, American Mathematical Society Colloquium Publications, vol. 18, 1934.

4. Sufficient conditions in the problem of Lagrange without assumptions of normalcy, T.A.M.S., vol. 37 (1935), pp. 147-160.

5. See Leighton [1].

Myers, S. B.

1. Sufficient conditions in the problem of the calculus of variations in $n$-space in parametric form and under general end conditions, T.A.M.S., vol. 35 (1933), pp. 746-760.

MYLLER, A.

1. Gewöhnliche Differentialgleichungen höherer Ordnung in ihrer Beziehung $z u$ den Integralgleichungen, Dissertation, Göttingen, 1906. Picone, M.

1. Sui valori eccezionali di un parametro da cui dipende un'equazione differenziale lineare ordinaria del secondo ordine, Annali della Reale Scuola Normale Superiore di Pisa, vol. 11 (1910), pp. 1-141. 
2. Sulle autosoluzioni e sulle formule di maggiorazione per gli integrali delle equazioni differenziali lineari ordinarie autoaggiunte, M.Z., vol. 28 (1928), pp. 519-555.

Plancherel, M.

1. Le passage à la limite des équations aux différences aux équations différentielles dans les problèmes aux limites, Bulletin des Sciences Mathématiques, vol. 47 (1923), pp. 153-160 and 170-177.

PORTER, M. B.

1. On the roots of functions connected by a linear recurrent relation of the second order, Ann. M., vol. 3 (1902), pp. 55-70.

PRÜFER, $\mathrm{H}$.

1. Neue Herleitung der Sturm-Liouvilleschen Reihenentwicklung stetiger Funktionen, M.A., vol. 95 (1926), pp. 499-518.

RADON, J.

1. Über die Oszillationsthecreme der konjugierten Punkte biem Probleme von Lagrange, Münchner Berichte, 1927, pp. 243-257.

2. Zum Problem von Lagrange, Abhandlungen aus dem Mathematischen Seminar der Hamburgischen Universität, vol. 6 (1928), pp. 273-299.

REID, W. T.

1. A boundary value problem associated with the calculus of variations, A.J.M., vol. 54 (1932), pp. 769-790.

2. Analogues of the Jacobi condition for the problem of Mayer in the calculus of variations, Ann. M., vol. 35 (1934), pp. 836-848.

3. Discontinuous solutions in the non-parametric problem of Mayer in the calculus of variations, A.J.M., vol. 57 (1935), pp. 69-93.

4. The theory of the second variation for the non-parametric problem of Bolza, A.J.M., vol. 57 (1935), pp. 573-586.

5. An integro-differential boundary problem, offered for publication to A.J.M.

6. A system of ordinary linear differential equations with two-point boundary conditions, offered for publication to T.A.M.S.

7. On Klein's oscillation theorem for boundary problems of the calculus of variations, for abstract see this Bulletin, vol. 43 (1937), p. 330.

RICHARDSON, R. G. D.

1. Das Jacobische Kriterium der Variationsrechnung und die Oszillationseigenschaften linearer Differentialgleichungen 2. Ordnung, M.A., vol. 68 (1910), pp. 279-304, and vol. 71 (1912), pp. 214-232.

2. Über die notwendigen und hinreichenden Bedingungen für das Bestehen eines Kleinschen Oszillationstheoreme, M.A., vol. 73 (1913), pp. 289-304.

3. Theorems of oscillation for two linear differential equations of the second order with two parameters, T.A.M.S., vol. 13 (1912), pp. 22-34.

4. Contributions to the study of oscillation properties of the solutions of linear differential equations of the second order, A.J.M., vol. 40 (1918), pp. 283-316.

5. A problem in the calculus of variations with an infinite number of auxiliary conditions, T.A.M.S., vol. 30 (1928), pp. 155-189.

SCHOENBERG, I. J.

1. See BLISs [3]. 
SCHUR, A.

1. Zur Entwicklung willkitrlicher Funktionen nach Lösungen von Systemen linearer Differentialgleichungen, M.A., vol. 82 (1921), pp. 213-236.

Severini, C.

1. Sopra alcuni sviluppi in serie di funzioni fondamentali, Atti della Reale Accademia dei Lincei, vol. 322 (1923), pp. 145-148 and 200-203; also Bollettino della Unione Matematica Italiana, vol. 2 (1923), pp. 173-176.

STARK, M. E.

1. A self-adjoint boundary value problem associated with a problem of the calculus of variations, Dissertation, Chicago, 1926.

STEKLOFF, W.

1. Solution générale du problème de développement d'une fonction arbitraire en séries suivant les fonctions fondamentales de Sturm-Liouville, Atti della Reale Accademia dei Lincei, vol. 192 (1910), pp. 490-496.

STERNBERG, W.

1. Die Entwicklung unstetiger Funktionen nach den Eigenfunktionen eines eindimensionalen Randwertproblems, M.Z., vol. 3 (1919), pp. 191-208.

STONE, M. H.

1. A comparison of the series of Fourier and Birkhoff, T.A.M.S., vol. 28 (1926), pp. 695-761.

2. The expansion problems associated with regular differential systems of the second order, T.A.M.S., vol. 29 (1927), pp. 826-844.

3. Irregular differential systems of order two and the related expansion problems, T.A.M.S., vol. 29 (1927), pp. 23-53.

StuRdivant, J. H.

1. Second order linear systems with summable coefficients, T.A.M.S., vol. 30 (1928), pp. 560-566.

STURM, C.

1. Mémoire sur les équations différentielles linéaires du second ordre, Journal de Mathématiques, vol. 1 (1836), pp. 106-186.

TAMARKIN, J. D.

1. Sur quelques points de la théorie des équations différentielles linéaires ordinaires et sur la généralization de la série de Fourier, Rendiconti del Circolo Matematico di Palermo, vol. 34 (1912), pp. 345-382.

2. Some general problems of the theory of ordinary linear differential equations and expansion of an arbitrary function in series of fundamental solutions, Petrograd, 1917, (in Russian), and M.Z., vol. 27 (1927), pp. 1-54.

TEMPLE, G.

1. The computation of characteristic numbers and characteristic functions, Proceedings of the London Mathematical Society, vol. 29 (1929), pp. 257-280. WALSH, J. L.

1. On the convergence of the Sturm-Liouville series, Ann. M., vol. 24 (1923), pp. 109-120.

Wheeler, Anna Pell

1. Linear ordinary self-adjoint differential equations of the second order A.J.M., vol. 49 (1927), pp. 309-320. 
WhYBURN, W. M.

1. Second-order differential systems with integral and k-point boundary contions, T.A.M.S., vol. 30 (1928), pp. 630-640.

2. Existence and oscillation theorems for non-linear differential systems of the second order, T.A.M.S., vol. 30 (1928), pp. 848-854.

3. On related difference and differential systems, A.J.M., vol. 51 (1929), pp. 265-286.

4. On self-adjoint ordinary differential equations of the fourth order, A.J.M., vol. 52 (1930), pp. 171-196.

WigGIN, E. P.

1. A boundary value problem of the calculus of variations, Dissertation, Chicago, 1936.

Yoshikawa, J.

1. Ein zweiparametriges Oszillationstheorem, Göttinger Nachrichten, 1910, pp. 586-594.

2. Dreiparametrige Randwertaufgaben, Göttinger Nachrichten, 1910, pp. 563-585.

\section{BOUNDARY PROBLEMS ASSOCIATED WITH DOUBLE AND MULTIPLE INTEGRALS OF THE CALCULUS OF VARIATIONS}

BuIss, G. A.

7. The Calculus of Variations, Multiple Integrals, Mimeographed notes of lectures delivered summer, 1933, at the University of Chicago.

HAAR, A.

2. Zur Variationsrechnung, Abhandlungen aus dem Mathematischen Seminar der Hamburgischen Universität, vol. 8 (1930), pp. 1-27.

Lichtenstein, L.

6. Untersuchungen ïber zweidimensionale reguläre Variationsprobleme, I, Monatshefte für Mathematik und Physik, vol. 28 (1917), pp. 3-51.

7. Zur Analysis der unendlich vielen Variablen, II, M.Z., vol. 3 (1919), pp. 127-160.

8. Untersuchungen über zweidimensionale reguläre Variationsprobleme, II, M.Z., vol. 5 (1919), pp. 26-51.

Picone, M.

3. Nuova condizione necessaria per un estremo di un integrale doppio, Atti della Reale Accademia dei Lincei, vol. 311 (1922), pp. 46-48 and 94-97. REID, W. T.

8. On boundary value problems associated with double integrals in the calculus of variations, A.J.M., vol. 54 (1932), pp. 791-801.

9. On the Jacobi condition for the double integral problem of the calculus of variations, for abstract see this Bulletin, vol. 42 (1936), p. 809.

Simmons, H. A.

1. The first and second variations of a double integral for the case of variable limits, T.A.M.S., vol. 28 (1926), pp. 235-251.

2. The first and second variations of an n-tuple integral in the case of variable limits, T.A.M.S., vol. 36 (1934), pp. 29-43.

The University of Chicago 